Editorial

\title{
TP53 and MTOR crosstalk to regulate cellular senescence
}

\author{
Lorenzo Galluzzi $^{1-3}$, Oliver Kepp ${ }^{1-3}$ and Guido Kroemer ${ }^{1,4-7}$
}

\author{
1 INSERM, U848, F-94805 Villejuif, France \\ 2 Institut Gustave Roussy, F-94805 Villejuif, France \\ 3 Université Paris -Sud, Paris 11, F-94805 Villejuif, France \\ 4 Metabolomics Platform, Institut Gustave Roussy, F-94805 Villejuif, France \\ 5 Centre de Recherche des Cordoliers, F-75005 Paris, France \\ 6 Pôle de Biologie, Hôpital Européen Georges Pompidou, AP-HP, F-75908 Paris, France \\ 7 Université Paris Descartes, Paris 5, F-75270 Paris, France
}

Key words: Aging; cancer; DNA damage response; nutlin; p21; rapamycin

Received: 09/16/10; accepted: 09/18/10; published on line: 09/18/10

Corresponding author: Guido Kroemer, PhD; E-mail: kroemer@orange.fr

Abstract: The full spectrum of activities of the tumor suppressor p53 (TP53) has not been completely elucidated yet. Recently, it was demonstrated that TP53 communicates with the metabolic regulator mechanistic target of rapamycin (MTOR) to determine whether stressed cells undergo cell death, reversible quiescence or irreversible senescence, thereby adding yet another level of complexity to the signaling network that emanate from TP53.

The oncosuppressor TP53, which is mutated or inactivated in more than $50 \%$ of all human neoplasms, is widely known for its ability to orchestrate a transcriptional stress response that can have multiple outcomes including senescence and cell death [1]. Thus, in reaction to a wide array of adverse conditions (e.g., DNA damage, oncogene deregulation), the TP53 protein gets stabilized by post-translational modifications and can transactivate cell cycle-arresting and/or lethal genes like CDKN1A (better known as $p 21^{C I P 1}$ ) and/or genes that code for pro-apoptotic members of the BCL2 protein family (e.g., $B A X$, $B B C 3$ ), respectively [1]. TP53 has also been shown to exert a number of extranuclear activities [2], including the induction of mitochondrial membrane permeabilization [3], the inhibition of autophagy [4] and the degradation of double-stranded RNA [5].

Recently, Demidenko and colleagues demonstrated that TP53 can convert CDKN1A-induced irreversible senescence into reversible quiescence through a mechanism that involves the central regulator of autophagy mechanistic target of rapamycin (MTOR) [6]. Similar to rapamycin (which is well known for its antiaging effects) [7], the accumulation of post-translational- ly unmodified, transactivation-proficient (but not transactivation-deficient) TP53 resulted in MTOR inhibition and senescence suppression [6]. These results suggest that TP53 can exert cell cycle-arresting and senescence-suppressing functions that can be uncoupled, at least in selected experimental settings.

Intrigued by these observations, Leontieva et al. have studied the response of immortalized WI-38 human lung fibroblasts to nutlin-3a (an inhibitor of the interaction between TP53 and its major negative regulator HDM2) and doxorubicin (a DNA damaging agent) [8]. In line with previous results [9], low concentrations of doxorubicin induced a prolonged cell cycle arrest and were highly efficient in driving WI-38 cells into senescence, which by definition is irreversible. On the contrary, both high doses of doxorubicin and nutlin-3a promoted quiescence, a reversible cell cycle arrest that can be overcome upon the removal of the triggering stimulus.

At the biochemical level, low doses of doxorubicin induced a modest accumulation of TP53 and CDKN1A transactivation, but did not inhibit the signaling cascade that emanate from MTOR. In this setting, the cell cycle 
arresting activity of TP53 prevailed and cells were driven into senescence [8]. On the other hand, both high doxorubicin concentrations and nutlin-3a provoked TP53 superinduction while inhibiting MTOR-mediated phosphorylation, a condition that resulted in reversible quiescence in spite of normal CDKN1A transactivation [8]. Notably, the co-administration of high doxorubicin and nutlin-3a led to TP53 hyperaccumulation, complete suppression of MTOR activity, poor transactivation of CDKN1A and cell death. These results indicate that the levels of TP53 and the activation status of the MTOR pathway are critical to determine whether, in nonapoptotic settings, CDKN1A will orchestrate an irreversible or a reversible cell cycle arrest [8].

Both TP53 and MTOR are known for their autophagymodulatory functions. While nuclear TP53 stimulates autophagy by transactivating several pro-autophagic genes, both cytoplasmic TP53 and MTOR tonically inhibit the autophagic flow [4]. Nutlin-3a-mediated senescence suppression (which proceeds through TP53 superinduction and MTOR inhibition) requires the transcriptional functions of TP53, implying that at least one, thus far elusive, TP53 target protein is responsible for TP53 senescence-suppressing functions [6]. One such TP53-responsive protein is sestrin 2, which can inhibit MTOR [10] and hence induce autophagy [11,
12]. However, the exact nature of the relevant $\mathrm{p} 53$ target(s) that regulate the switch between senescence and quiescence remains elusive.

Based on the fascinating results obtained by Leontieva et al. [8], it can be speculated that nuclear TP53 might simultaneously transactivate the cell cycle-arresting factor CDKN1A and one or more hitherto unidentified anti-senescence (and perhaps pro-autophagic?) protein(s) that would operate similar to rapamycin, through the inhibition of MTOR. How would then the senescence-inducing activity of TP53 prevail over TP53-mediated senescence suppression (and vice versa)? As a possibility, the promoter of CDKN1A might display a high affinity for TP53, while the promoter of the TP53 target that suppresses MTOR activity might require high TP53 concentrations for efficient transactivation. This hypothesis takes into consideration the fact that CDKN1A is induced at similar levels by both low and high doses of doxorubicin, as well as by low and high concentrations of nutlin-3a [8]. In this scenario, the accumulation of TP53 beyond a low threshold would activate CDKN1A-mediated senescence (Figure 1A), whereas high levels of TP53 would be required for the ignition of a senescence-suppressing program that (once started) would always prevail over the effects of CDKN1A (Figure 1B).

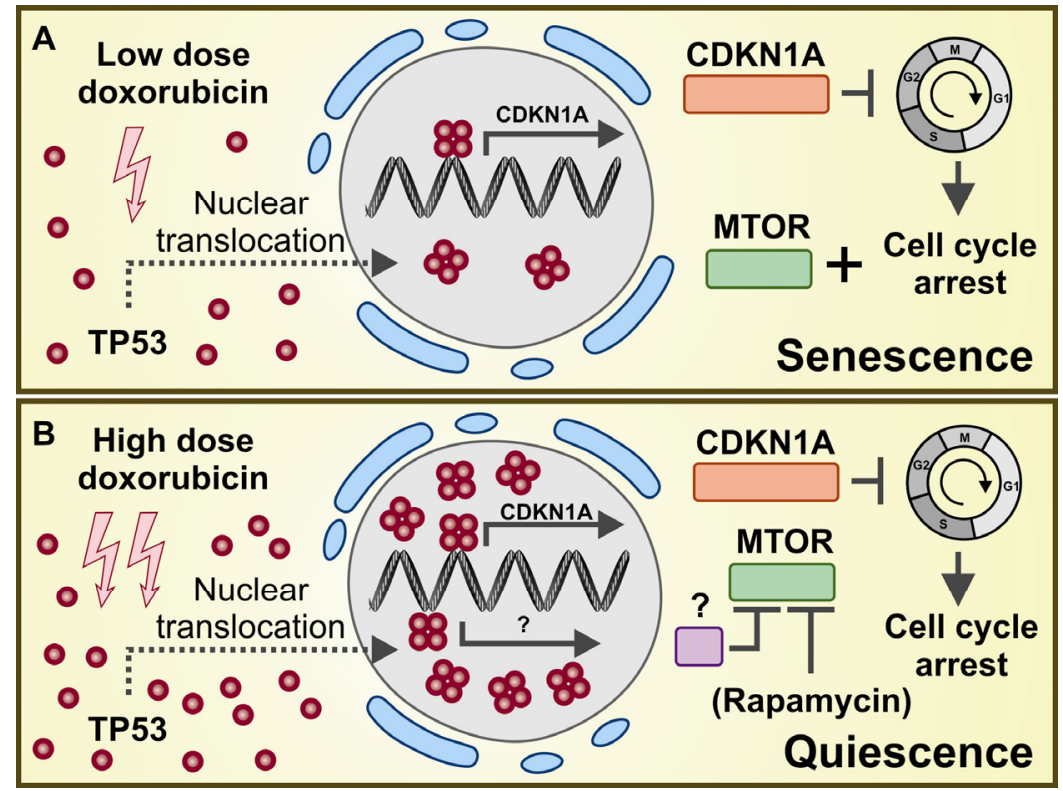

Figure 1. TP53 levels determine whether CDKN1A will orchestrate irreversible senescence or quiescence. (A) Low doses of doxorubicin are sufficient to trigger TP53-mediated transactivation of the cell cycle-arresting protein CDKN1A. Under conditions in which the MTOR pathway is active, prolonged cell cycle arrest results in irreversible senescence. (B) High doxorubicin concentrations (or nutlin-3a alone or in combination with low doses of doxorubicin) not only drive TP53-mediated CDKN1A transactivation but might also result in the induction of one (or more) senescence-suppressing factors. In this scenario, MTOR activity is suppressed and CDKN1A-mediated cell cycle arrest is reversible (quiescence). By pharmacologically inhibiting MTOR, rapamycin also exerts senescence-suppressing functions. 
Further experimental work is urgently required to confirm or invalidate this hypothesis. Beyond these unresolved details, the work by Leontieva et al. added yet another important piece to the ever-growing TP53 puzzle.

\section{ACKNOWLEDGEMENTS}

GK is supported by the Ligue Nationale contre le Cancer (Equipe labellisé), Agence Nationale pour la Recherche (ANR), European Commission (Active p53, Apo-Sys, ChemoRes, ApopTrain), Fondation pour la Recherche Médicale (FRM), Institut National du Cancer (INCa), Cancéropôle Ile-de-France and AXA Research Fund. LG and OK are supported by the Apo-Sys consortium of the European Union and Association pour la Recherche sur le Cancer (ARC), respectively.

\section{REFERENCES}

1. Levine AJ, Oren $M$. The first 30 years of p53: growing ever more complex. Nat Rev Cancer. 2009; 9: 749-758.

2. Green DR, Kroemer G. Cytoplasmic functions of the tumour suppressor p53. Nature. 2009; 458: 1127-1130.

3. Morselli E, Galluzzi L, Kroemer G. Mechanisms of p53mediated mitochondrial membrane permeabilization. Cell Res. 2008; 18: 708-710.

4. Maiuri MC, Galluzzi L, Morselli E, Kepp O, Malik SA, Kroemer G. Autophagy regulation by p53. Curr Opin Cell Biol. 2010; 22: 181-185.

5. Galluzzi L, Kepp O, Kroemer G. A new role for cytoplasmic p53: Binding and destroying double-stranded RNA. Cell Cycle. 2010; 9: 13: 2491-2492.

6. Demidenko ZN, Korotchkina LG, Gudkov AV, Blagosklonny MV. Paradoxical suppression of cellular senescence by p53. Proc Natl Acad Sci U S A. 2010; 107: 9660-9664.

7. Madeo F, Tavernarakis N, Kroemer G. Can autophagy promote longevity? Nat Cell Biol. 2010; 12: 842-846.

8. Leontieva OV, Gudkov AV, Blagosklonny MV. Weak p53 permits senescence during cell cycle arrest. Cell Cycle. 2010: 9: in press.

9. Chang BD, Broude EV, Dokmanovic M, Zhu H, Ruth A, Xuan Y, et al. A senescence-like phenotype distinguishes tumor cells that undergo terminal proliferation arrest after exposure to anticancer agents. Cancer Res. 1999; 59: 3761-3767.

10. Budanov AV, Karin M. p53 target genes sestrin 1 and sestrin 2 connect genotoxic stress and mTOR signaling. Cell. 2008; 134: 451-460.

11. Criollo A, Dessen P, Kroemer G. DRAM: a phylogenetically ancient regulator of autophagy. Cell Cycle. 2009; 8: 2319-2320.

12. Maiuri MC, Malik SA, Morselli E, Kepp O, Criollo A, Mouchel $\mathrm{PL}$, et al. Stimulation of autophagy by the p53 target gene Sestrin 2. Cell Cycle. 2009; 8: 1571-1576. 